\title{
Multicentric reticulohistiocytosis in the course of undifferentiated connective tissue disease
}

\author{
Karolina Niklas ${ }^{1}$, Arkadiusz Niklas ${ }^{2}$, Mariusz Puszczewicz ${ }^{1}$ \\ ${ }^{1}$ Department of Rheumatology and Internal Diseases, Poznan University of Medical Sciences, Poznan, Poland \\ ${ }^{2}$ Department of Hypertension, Angiology and Internal Medicine, Poznan University of Medical Sciences, Poznan, Poland \\ Adv Dermatol Allergol 2019; XXXVI (5): 646-647 \\ DOI: https://doi.org/10.5114/ada.2019.83500
}

Multicentric reticulohistiocytosis $(\mathrm{MRH})$ is a rare disease with unknown aetiology, characterized by the reactive proliferation of histiocytes in the skin, mucous membrane, subcutaneous tissue, synovium, and the bones. Its clinical symptoms are skin changes with red, brown, or pink papules and nodules. They can be located on the hands, face, trunk, or mucous membranes. The skin changes are typically accompanied with symmetrical arthritis, primarily of minor hand joints, which may lead to joint destruction and in extreme cases, to arthritis mutilans changes. MRH is typically related to rheumatoid diseases or considered to be a paraneoplastic syndrome $[1,2]$.

Here, we present the case of a woman, aged 39 years, with skin changes observed for the previous 5 months in the form of reddish brown painless nodules located near the minor hand joints, typically at the metacarpophalangeal and proximal interphalangeal joints and on the auricles. These skin changes were accompanied by pain and swelling in the metacarpophalangeal and proximal interphalangeal joints. To determine the diagnosis, a specimen was obtained from the skin changes for a histopathological examination; the obtained image corresponded to MRH. The following treatment was started: methylprednisolone $4 \mathrm{mg}$ every other day and sulfasalazine $3 \mathrm{~g}$ /day in divided doses. Further, the patient was referred to our department. On admission to the Rheumatology Department, the described skin changes were still present (Figure 1), while the pain and swelling of the joints had decreased slightly after the applied treatment. The laboratory examination revealed normal erythrocyte sedimentation rate (ESR), normal C-reactive protein (CRP) concentration, benign anaemia $(\mathrm{Hb} 10.7 \mathrm{~g} / \mathrm{dl})$, presence of rheumatoid factor (RF), antibodies against cyclic citrullinated peptide (aCCP), and antinuclear antibodies of titre $1: 1280$ with the presence of the SSA and SSB antibodies. The levels of tumour markers (CEA, AFP, Ca125, and
CA19.9) did not exceed the reference values. The chest $X$-ray did not exhibit changes in the organs. An ultrasonography of the abdominal organs did not reveal any changes. Hand X-ray did not reveal any changes in the osseous structure (Figure 2). Because of the serological profile, despite the absence of symptoms both in the eyes and the oral cavity, Schirmer's test was performed; a positive result was obtained (right eye $10 \mathrm{~mm}$, left eye $10 \mathrm{~mm}$ ). Undifferentiated connective tissue disease (UCTD) was diagnosed, with the need for further observation to determine whether the patient was suffering from Sjögren's syndrome. The treatment was modified: sulfasalazine was discontinued, the methylprednisolone dose was increased to $4 \mathrm{mg} /$ day, and chloroquine was introduced at a dose of $250 \mathrm{mg} /$ day. Hospitalization in 6 months' time was planned.

In the analysed literature, we could find only one case of MRH co-occurring with UCTD [3]. West et al. described a female patient, aged 47 years, who was additionally diagnosed with usual interstitial pneumonia (UIP). No changes in the respiratory system were found in our patient. Our patient may develop Sjögren's syndrome (SS); however, currently, no subjective symptoms associated with the dryness of the eyes or the oral cavity have been observed and the patient does not meet diagnostic criteria. The existing literature contains several cases of the co-occurrence of MRH and SS. In most of these cases, skin changes are accompanied by arthritis, typically of a destructive nature. Ben Abdelghani et al. have even described that MHR may be incorrectly diagnosed as rheumatoid arthritis (RA) [4]. In the case of our patient, we have not observed any changes in the osseous structure, despite the presence of RF and ACCP and the clinical symptoms of arthritis, in contrast to the previously described case of MRH co-occurring with UCTD, where joint destructive lesions were observed despite a medical history of only several months. Moreover, in the case

Address for correspondence: Karolina Niklas MD, PhD, Department of Rheumatology and Internal Diseases, Poznan University of Medical Sciences, 135/147 28 Czerwca 1956 r. St, 61-545 Poznan, Poland, phone/fax: +48 6183103 17, e-mail: karolina_niklas@vp.pl Received: 2.07.2018, accepted: 11.07.2018. 


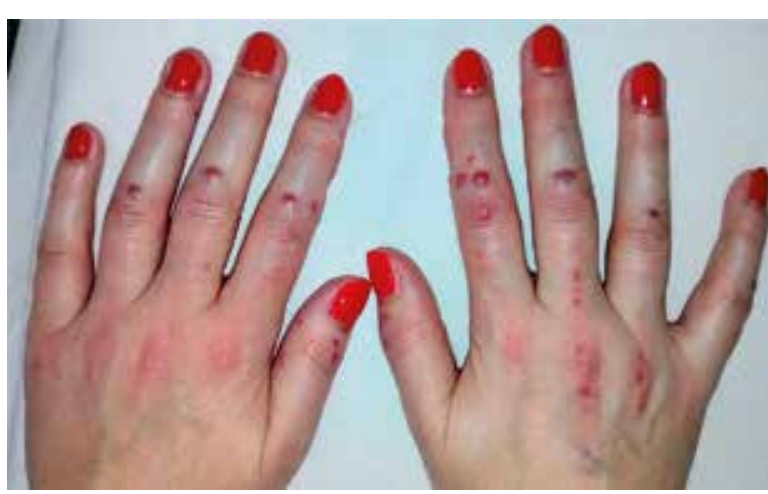

Figure 1. Skin changes located on the hands

of subclinical Sjögren's syndrome accompanying MRH, described by Shiokawa et al., erosive changes in the minor hand joints were observed and RA was originally suspected [5]. Destructive arthritis was also observed in the case of co-occurrence of MRH and SS in the case described by Carey et al. [6]. Moreover, MRH may accompany other systemic connective tissue disorders. An interesting case of the co-occurrence of $\mathrm{MRH}, \mathrm{SS}$, and systemic sclerosis was described by Takahashi et al. [7]. Further, MRH was determined in the course of systemic lupus erythematosus, polymyositis, and dermatomyositis $[1,8,9]$. The incidence of MRH was also described in the cases of other autoimmune diseases, such as hypothyroidism and diabetes [1].

In the case of a patient with $\mathrm{MRH}$, the relationship with cancer must be excluded. A relationship of MHR with leukemia, lymphoma, lung cancer, ovary cancer, endometrium cancer, breast cancer, stomach cancer, colorectal cancer, melanoma, and mesothelioma has been described $[1,3,10]$. In our patient, we were unable to determine characteristics that could suggest a cancer in the clinical examination and in the diagnostic imaging. Further, the cancer marker results remained within the limits of the reference values.

Our case appears to be the second co-occurrence of MRH and UCTD discussed in the literature. Moreover, we did not find any destructive lesions in the joints of our patient. This could perhaps be attributed to the very early treatment with disease-modifying drugs.

\section{Conflict of interest}

The authors declare no conflict of interest.

\section{References}

1. Toz B, Büyükbabani N, Inanç M. Multicentric reticulohistiocytosis: rheumatology perspective. Best Pract Res Clin Rheumatol 2016; 30: 250-60.

2. Swierkot J, Lewandowicz-Uszyńska A, Bogunia-Kubik K. Paraneoplastic syndromes and rheumatic diseases. Postep Hig Med Dosw 2014; 68: 944-54.

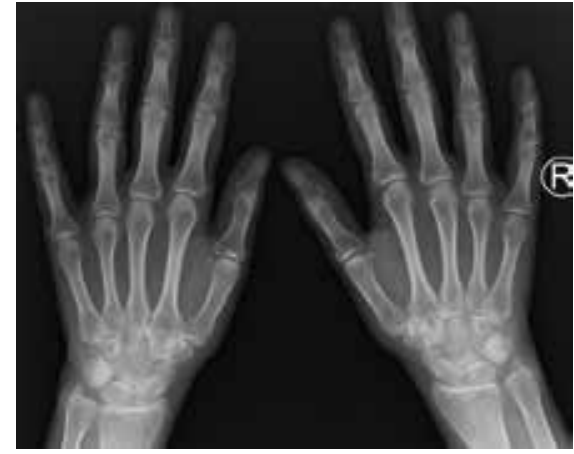

Figure 2. X-ray of the hands of our patient without any changes in the osseous structure

3. West KL, Sporn T, Puri PK. Multicentric reticulohistiocytosis: a unique case with pulmonary fibrosis. Arch Dermatol 2012; 148: 228-32.

4. Ben Abdelghani K, Mahmoud I, Chatelus E, et al. Multicentric reticulohistiocytosis: an autoimmune systemic disease? Case report of an association with erosive rheumatoid arthritis and systemic Sjogren syndrome. Joint Bone Spine 2010; 77: 274-6.

5. Shiokawa S, Shingu M, Nishimura M, et al. Multicentric reticulohistiocytosis associated with subclinical Sjögren's syndrome. Clin Rheumatol 1991; 10: 201-5.

6. Carey RN, Blotzer JW, Wolfe ID, et al. Multicentric reticulohistiocytosis and Sjögren's syndrome. J Rheumatol 1985; 12: 1193-5.

7. Takahashi M, Mizutani H, Nakamura Y, Shimizu M. A case of multicentric reticulohistiocytosis, systemic sclerosis and Sjögren syndrome. J Dermatol 1997; 24: 530-4.

8. Saito K, Fujii K, Awazu Y, et al. A case of systemic lupus erythematosus complicated with multicentric reticulohistiocytosis (MRH): successful treatment of $\mathrm{MRH}$ and lupus nephritis with cyclosporin A. Lupus 2001; 10: 129-32.

9. Hsiung SH, Chan EF, Elenitsas R, et al. Multicentric reticulohistiocytosis presenting with clinical features of dermatomyositis. J Am Acad Dermatol Suppl 2003; 48 (2 Suppl): S11-4.

10. Luz FB, Gaspar TAP, Kalil-Gaspar N, Ramos-e-Silva M. Multicentric reticulohistiocytosis. J Eur Acad Dermatol Venereol 2001; 15: 524-31. 